\title{
Identifying Patient Profiles Suitable for Cognitive Behavior Therapy: The Role of Psychological Mindedness
}

Sir,

Psychological interventions, such as cognitive behavior therapy (CBT), have robust evidence for efficacy in many psychiatric and even some nonpsychiatric disorders. ${ }^{[1]}$ Notwithstanding this considerable evidence base, cultural variations in receptiveness and efficacy of psychotherapeutic techniques have gained a lot of clinical and research attention.

CBT differs from the other major form of therapy, namely pharmacotherapy, in many aspects. First, the former is more active, collaborative and requires the patient and therapist to jointly invest more time and effort. Second, CBT usually involves a variety of homework assignments, and often, the family members are also called upon to play the role of a cotherapist. Factoring in these differences, it is clear that CBT or similar directive psychotherapies may not be everyone's cup of tea. Further, in the Indian culture, introspection and talking about one's emotional troubles are often frowned upon. ${ }^{[2]}$ It, therefore, becomes incumbent upon clinicians to identify patient profiles suitable for CBT to optimize chances of response. One of the important factors that have been posited to play a role in acceptability and treatment outcomes with CBT is the concept of psychological mindedness.

Psychological mindedness is defined as person's capacity for self-examination, self-reflection, introspection, and personal insight. ${ }^{[3]}$ It includes an ability to recognize meanings that underlie overt words and actions, to identify and appreciate emotional nuance and complexity, to analyze what underlies overt words and actions to recognize the links between past and present, and to insight into one's own and others' motives and intentions. Psychologically minded people tend to have higher insight into their illness.

There is some evidence linking higher psychological mindedness and favorable outcomes in both short-term supportive and interpretive psychotherapies. ${ }^{[4]}$ 
However, trials which have investigated psychological mindedness as a moderator for treatment outcomes in CBT did not find clear evidence of effect modification, ${ }^{\left[{ }^{5}\right]}$ though the authors of this trial point out that sample sizes were inadequate for testing interactions between psychological mindedness and therapy outcomes. Lower dysfunctional attitudes have been found to mediate outcomes with both pharmacotherapy and psychotherapy in chronic depression. ${ }^{[6]}$ However, there is a dearth of studies that have directly examined the utility of metacognitive constructs such as psychological mindedness in moderating response to CBT.

To facilitate such studies yielding valid results, we need to further operationalize the construct of psychological mindedness in observational and interventional studies of CBT. The effect of psychological mindedness on the acceptability of CBT-based psychotherapeutic interventions in diverse cultural contexts may be looked into. We suggest that future studies should examine the moderating role of psychological mindedness by checking the interaction between psychological mindedness and CBT versus one or two comparator treatments, in the context of a robust randomized controlled design. This will help us conclude the role of psychological mindedness in moderating CBT-related outcomes. This will also pave the way for personalizing CBT approaches and optimize treatment outcomes.

\section{Financial support and sponsorship}

Nil.

\section{Conflicts of interest}

There are no conflicts of interest.

\section{Sivapriya Vaidyanathan, Vikas Menon, Siddharth Sarkar ${ }^{1}$}

Department of Psychiatry, Jawaharlal Institute of Postgraduate Medical Education and Research, Puducherry, ${ }^{1}$ Department of Psychiatry and National Drug Dependence Treatment Center, All India Institute of Medical Sciences, New Delhi, India
Address for correspondence: Dr. Vikas Menon, Department of Psychiatry, Jawaharlal Institute of Postgraduate Medical Education and Research, Dhanvantri Nagar, Puducherry - 605 006, India. E-mail:drvmenon@gmail.com

\section{REFERENCES}

1. Chand SP, Whitten RA. Cognitive Behavior Therapy. In: Treasure Island (FL). StatPearls Publishing; 2017. Available from: http://www.ncbi.nlm.nih.gov/books/NBK470241/. [Last accessed on 2018 Feb 17].

2. Gautam S, Jain N. Indian culture and psychiatry. Indian J Psychiatry 2010;52:S309-13.

3. Hall JA. Psychological-mindedness: A conceptual model. Am J Psychother 1992;46:131-40.

4. McCallum M, Piper WE, Ogrodniczuk JS, Joyce AS. Relationships among psychological mindedness, alexithymia and outcome in four forms of short-term psychotherapy. Psychol Psychother 2003;76:133-44.

5. Wiles N, Thomas L, Abel A, Barnes M, Carroll F, Ridgway N, et al. Clinical effectiveness and cost-effectiveness of cognitive behavioural therapy as an adjunct to pharmacotherapy for treatment-resistant depression in primary care: The CoBalT randomised controlled trial. Health Technol Assess 2014;18:1-167, vii-viii.

6. Shankman SA, Campbell ML, Klein DN, Leon AC, Arnow BA, Manber R, et al. Dysfunctional attitudes as a moderator of pharmacotherapy and psychotherapy for chronic depression. J Psychiatr Res 2013;47:113-21.

This is an open access journal, and articles are distributed under the terms of the Creative Commons Attribution-NonCommercial-ShareAlike 4.0 License, which allows others to remix, tweak, and build upon the work non-commercially, as long as appropriate credit is given and the new creations are licensed under the identical terms.

\begin{tabular}{|l|l|}
\hline \multicolumn{2}{|c|}{ Access this article online } \\
\hline Quick Response Code: & Website: \\
\hline
\end{tabular}

How to cite this article: Vaidyanathan S, Menon V, Sarkar S. Identifying patient profiles suitable for cognitive behavior therapy: The role of psychological mindedness. J Neurosci Rural Pract 2018;9:654-5.

(C) 2018 Journal of Neurosciences in Rural Practice | Published by Wolters Kluwer - Medknow 